\title{
SUB-RESOLUTION MAXIMUM-LIKELIHOOD BASED LOCALIZATION OF FLUORESCENT NANOPARTICLES IN THREE DIMENSIONS
}

\author{
François Aguet, Dimitri Van De Ville, and Michael Unser \\ Biomedical Imaging Group \\ Ecole Polytechnique Fédérale de Lausanne
}

\begin{abstract}
Several recent studies have shown that fluorescent particles can be localized with an accuracy that is well beyond traditional resolution limits. Using a theoretical model of the image formation process that accounts for possible sources of noise, Cramér-Rao bounds have been used to define the theoretical limits. A crucial influence on these bounds is the mismatch of refractive indices that is usually present between immersion medium and specimen. This results in an axially shift-variant point spread function, meaning that the bounds change as a function of the particle's position in the zdirection. We investigate the theoretical bounds for this shiftvariant model, and propose a maximum-likelihood estimator for the particle position in 3D (XYZ position). Using this estimator, sub-resolution localization at the nanometer scale is demonstrated on experimental data. The results provide optimal conditions for particle tracking and localization experiments.
\end{abstract}

Index Terms - Fluorescence, Microscopy, Maximum likelihood estimation, Optical transfer functions

\section{INTRODUCTION}

New markers such as fluorescent proteins and quantum dots have become indispensable tools for dynamic studies of molecular interactions in living cells [1]. An essential aspect of these studies lies in the accuracy with which an individual fluorescent marker can be spatially localized from its image through a microscope. The size of these markers is typically of the order of $10 \mathrm{~nm}$, which is significantly smaller than the optical resolution of a fluorescence microscope. Efforts are currently being made both in instrumentation and image processing to surpass this resolution limit. For example, by controlling the excitation of the sample in a precise way, techniques such as STED microscopy have led to significantly improved lateral and axial resolutions [2]. Similarly, recent work on computational approaches to localization yielded results with an accuracy at the nanometer scale $[3,4,5]$. Fluorophores can be assimilated to point sources, and therefore,

This work was supported by the Swiss National Science Foundation under grant 200020-109415, as well as by the Hasler foundation. localization of a particle essentially amounts to fitting a model of the microscope's point spread function (PSF) to the acquired image(s) of the particle, which may be corrupted by various types of noise. Betzig et al. recently proposed a technique which combines selective excitation of individual fluorophores with a localization algorithm based on a Gaussian fit, and obtained results with a resolution of a few nanometers, rivaling electron microscopy [6]. Several studies have established the theoretical lower bounds on the attainable localization accuracy in the plane [4] and along the optical axis [5], using Cramér-Rao bounds. Here, we extend our previous analysis on axial localization to a complete framework for the localization of fluorophores in 3-D. In particular, we focus on the influence of the shift-variant PSF on the localization bounds and propose an estimator that reaches these bounds.

\section{IMAGE FORMATION MODEL}

We first introduce the PSF model used in our work. Subsequently we formulate a general model for acquisition noise and briefly discuss the effects of discretization and pixelation by the CCD array.

\subsection{PSF model}

Due to design constraints of modern microscope optics, a mismatch between the refractive indices of the immersion medium and the specimen inevitably leads to spherical aberrations that are especially significant for high NA objectives. These aberrations vary as a function of the source's depth within the specimen, resulting in a shift-variant PSF along the optical axis. This phenomenon is most accurately characterized by the vectorial models proposed by Hell et al. [7] and Török et al. [8]. They are computationally costly, however, and offer no significant gain in accuracy for the application discussed here with respect to the scalar model proposed by Gibson and Lanni [9]. This model takes the general form

$$
h\left(\mathbf{x}, \mathbf{x}_{p} ; \mathbf{p}\right)=\left|A \int_{0}^{1} e^{i W\left(\rho, \mathbf{x}, \mathbf{x}_{p} ; \mathbf{p}\right)} J_{0}(k r \mathrm{NA} \rho) \rho \mathrm{d} \rho\right|^{2},
$$

where $\mathbf{x}=(x, y, z)$ is a point on the image plane in object space, $\mathbf{x}_{p}=\left(x_{p}, y_{p}, z_{p}\right)$ are the coordinates of the particle, 
$\mathbf{p}=(\mathrm{NA}, \mathbf{n}, \mathbf{t})$ is a parameter vector containing the optical parameters of the system: numerical aperture (NA), refractive index (n) and thickness (t) of the immersion / coverslip / specimen layers, $r=\sqrt{\left(x-x_{p}\right)^{2}+\left(y-y_{p}\right)^{2}}, A$ is a constant amplitude, and $J_{\tau}$ denotes the Bessel function of the first kind of order $\tau$. For ease of notation, all coordinates are expressed in object space, which implies that we consider the image space to be demagnified and projected into object space. For further details the reader is referred to $[9,5]$.

The phase term $W\left(\rho, \mathbf{x}, \mathbf{x}_{p} ; \mathbf{p}\right)$ is proportional to the optical path difference between the actual and ideal imaging conditions. When no index mismatches occur, it reduces to

$$
W\left(\rho, \mathbf{x}, \mathbf{x}_{p} ; \mathbf{p}\right)=k \Delta z \sqrt{n_{i}^{2}-\mathrm{NA}^{2} \rho^{2}}
$$

which is the standard defocus model (linear approximation of this expression yields the classical Born \& Wolf phase aberration term). Note that for $\Delta z=0$, Eq. (1) further reduces to the familiar Airy function.

\subsection{Noise model}

The dominant kinds of noise in fluorescence microscopy are shot noise, background noise due to autofluorescence of the sample, and read-out noise. Whereas the first two obey Poisson statistics, the latter is Gaussian distributed, which calls for an additive noise model. The Poisson distribution rapidly converges towards a Gaussian with equal mean and variance, given that the latter is large enough (this is usually considered the case when $\sigma^{2}>10$, and is a valid assumption for the read-out noise measured in our experiments). Taking this into account, we establish a general noise model where we formulate the expected photon count $\bar{q}\left(\mathbf{x}, \mathbf{x}_{p} ; \mathbf{p}\right)$ corresponding to a point $\mathbf{x}$ in object space as

$$
\bar{q}\left(\mathbf{x}, \mathbf{x}_{p} ; \mathbf{p}\right)=c \cdot\left(h\left(\mathbf{x}, \mathbf{x}_{p} ; \mathbf{p}\right)+\sigma_{b}^{2}\right)
$$

where $c$ is a conversion factor and $\sigma_{b}^{2}$ is the variance (in intensity) of the read-out noise. To simplify the notation, we will omit the argument $\left(\mathbf{x}, \mathbf{x}_{p} ; \mathbf{p}\right)$ when it is clear from the context. by

The probability of detecting $q$ photons at $\mathbf{x}$ is then given

$$
P\left(q\left(\mathbf{x}, \mathbf{x}_{p} ; \mathbf{p}\right)\right)=\frac{e^{-\bar{q}(\bar{q})^{q}}}{q !}
$$

\subsection{Pixelation}

The effects of pixelation of the detector have been extensively covered by Ober et al. [4]. In our notation, it is assumed hereinafter that a point of observation $\mathrm{x}$ represents a pixel on the $\mathrm{CCD}$, and thus that, when appropriate, functions of $\mathrm{x}$ incorporate integration over the pixel's area (pixels are assumed to be contiguous and non-overlapping).

\section{THEORETICAL BOUNDS}

We proceed with an analysis of the localization accuracy attainable with an estimator based on the proposed image formation model. A limit on the localization accuracy can be obtained by means of the Cramér-Rao bound (CRB), which is a theoretical lower bound on the variance of an arbitrary unbiased estimator. For multiple parameters, this bound is given by the diagonal of the inverse of the Fisher information matrix. Under the assumption that the PSF is radially symmetric, the Fisher information matrix is diagonal and the CRBs for the coordinates of the particle's position $\mathbf{x}_{p}$ are given by

$$
\operatorname{Var}\left(\hat{x}_{p}\right) \geq 1 /-E\left[\frac{\partial^{2}}{\partial x^{2}} \ln \prod_{\mathbf{x} \in \mathcal{S}} P\left(\bar{q}\left(\mathbf{x}, \mathbf{x}_{p} ; \mathbf{p}\right)\right)\right]
$$

where $\mathcal{S}$ is the set of pixels in the acquisition, and where $\hat{x}_{p}$ is an unbiased estimator of the particle's $x$-coordinate, with homologous expressions for the bounds on $y$ and $z$. Evaluating the bounds for our image formation model yields

$$
\operatorname{Var}\left(\hat{x}_{p}\right) \geq 1 / \sum_{\mathbf{x} \in \mathcal{S}} \frac{\left(\frac{\partial}{\partial x} \bar{q}\left(\mathbf{x}, \mathbf{x}_{p} ; \mathbf{p}\right)\right)^{2}}{\bar{q}\left(\mathbf{x}, \mathbf{x}_{p} ; \mathbf{p}\right)},
$$

again with homologous expressions for $\operatorname{Var}\left(\hat{y}_{p}\right)$ and $\operatorname{Var}\left(\hat{z}_{p}\right)$. The partial derivatives of the PSF are given by

$$
\frac{\partial h\left(\mathbf{x}, \mathbf{x}_{p} ; \mathbf{p}\right)}{\partial x_{p}}=2 k|A|^{2} \mathrm{NA} \frac{x-x_{p}}{r}\left(c_{1,2} c_{0,1}+s_{1,2} s_{0,1}\right),
$$

with an equivalent expression for $\frac{\partial h\left(\mathbf{x}, \mathbf{x}_{p} ; \mathbf{p}\right)}{\partial y_{p}}$, where

$$
\begin{aligned}
& c_{\tau_{1}, \tau_{2}}\left(\mathbf{x}, \mathbf{x}_{p} ; \mathbf{p}\right)=\int_{0}^{1} \cos \left(W\left(\rho, \mathbf{x}, \mathbf{x}_{p} ; \mathbf{p}\right)\right) J_{\tau_{1}}(k r \mathrm{NA} \rho) \rho^{\tau_{2}} \mathrm{~d} \rho \\
& s_{\tau_{1}, \tau_{2}}\left(\mathbf{x}, \mathbf{x}_{p} ; \mathbf{p}\right)=\int_{0}^{1} \sin \left(W\left(\rho, \mathbf{x}, \mathbf{x}_{p} ; \mathbf{p}\right)\right) J_{\tau_{1}}(k r \mathrm{NA} \rho) \rho^{\tau_{2}} \mathrm{~d} \rho .
\end{aligned}
$$

The partial derivative with respect to $z_{p}$ is given in [5, Appendix].

\section{MAXIMUM-LIKELIHOOD ESTIMATOR}

We now introduce an estimator for a particle's position that is optimal in the sense that it reaches the theoretical bounds stated above. The probability of observing a given spatial distribution $q\left(\mathbf{x}, \mathbf{x}_{p} ; \mathbf{p}\right)$ of photons emitted by a source located at $\mathbf{x}_{p}$ is given by

$$
\prod_{\mathbf{x} \in \mathcal{S}} P\left(q\left(\mathbf{x}, \mathbf{x}_{p} ; \mathbf{p}\right)\right)
$$

By maximizing the likelihood of Eq. (9) with respect to $x_{p}$ we obtain the basis for our estimator:

$$
\frac{\partial}{\partial x} \ln \prod_{\mathbf{x} \in \mathcal{S}} P(q)=\sum_{\mathbf{x} \in \mathcal{S}} \frac{\partial \bar{q}}{\partial x}\left(\frac{q}{\bar{q}}-1\right) \equiv 0 .
$$


Since there is no closed-form solution for $x_{p}$ in the above expression, we take the first-order Taylor approximation of the maximum-likelihood function around an estimate $\hat{x}_{p}$ of $x_{p}$, which gives us the following iterative expression:

$$
\hat{x}_{p}^{(m+1)}=\hat{x}_{p}^{(m)}-\frac{\sum_{\mathbf{x} \in \mathcal{S}}\left(\frac{\partial \bar{q}}{\partial x}\left(\frac{q}{\bar{q}}-1\right)\right)}{\sum_{\mathbf{x} \in \mathcal{S}}\left(\frac{\partial^{2} \bar{q}}{\partial x^{2}}\left(\frac{q}{\bar{q}}-1\right)-\left(\frac{\partial \bar{q}}{\partial x}\right)^{2} \frac{q}{(\bar{q})^{2}}\right)},
$$

where $m$ denotes the iteration. The second partial derivative of the PSF with respect to $x_{p}$ is given by

$$
\begin{aligned}
& \frac{\partial^{2} h\left(\mathbf{x}, \mathbf{x}_{p} ; \mathbf{p}\right)}{\partial x_{p}^{2}}=\left(\frac{2\left(x-x_{p}\right)}{r^{2}}-\frac{1}{x-x_{p}}\right) \frac{\partial h\left(\mathbf{x}, \mathbf{x}_{p} ; \mathbf{p}\right)}{\partial x_{p}} \\
& +2 k^{2}|A|^{2} \mathrm{NA}^{2} \frac{\left(x-x_{p}\right)^{2}}{r^{2}}\left(c_{1,2}^{2}+s_{1,2}^{2}-c_{0,3} c_{0,1}-s_{0,3} s_{0,1}\right) .
\end{aligned}
$$

As before, the expression for $\frac{\partial^{2} h\left(\mathbf{x}, \mathbf{x}_{p} ; \mathbf{p}\right)}{\partial y_{p}^{2}}$ is identical up to the partial derivative, and the second partial derivative with respect to $z_{p}$ is given in our earlier work [5, Appendix].

The proposed linearization holds only locally and thus requires an adequate initialization. This can be obtained by evaluating the normalized cross-correlation between the acquisition and a precomputed set of 3D PSFs corresponding to the range of possible particle positions:

$$
\hat{\mathbf{x}}_{p}=\arg \max _{\mathbf{x}_{p} \in \mathcal{S}} \frac{\sum_{\mathbf{x} \in \mathcal{S}}\left(q-\mu_{q}\right)\left(\bar{q}-\mu_{\bar{q}}\right)}{\sqrt{\sum_{\mathbf{x} \in \mathcal{S}}\left(q-\mu_{q}\right)^{2} \sum_{\mathbf{x} \in \mathcal{S}}\left(\bar{q}-\mu_{\bar{q}}\right)^{2}}},
$$

where $\mu_{q}$ and $\mu_{\bar{q}}$ are the mean pixel values in acquisitions and model, respectively. The estimation can be stopped when the absolute value of the update step becomes smaller than the CRB by an order of magnitude, after which further refining the estimate becomes irrelevant.

\section{RESULTS}

We now provide some examples of our estimator and its performance relative to the $\mathrm{CRB}$ for some specific situations: first in simulation and then on experimental data. The acquisition parameters are the same for all examples provided, and are taken from the experiments, which were carried out on a Zeiss Axioplan 2 microscope equipped with an AxioCam CCD with pixels of size $6.45 \times 6.45 \mu \mathrm{m}^{2}$. The objective used is a Zeiss Plan-Apochromat 1.4 NA oil-immersion objective. The test sample consisted of $100 \mathrm{~nm}$-diameter beads fluorescing at $430 \mathrm{~nm}$, deposited onto a microscope slide and embedded in a solid mounting medium of refractive index 1.46. The immersion oil has a refractive index of 1.515 .
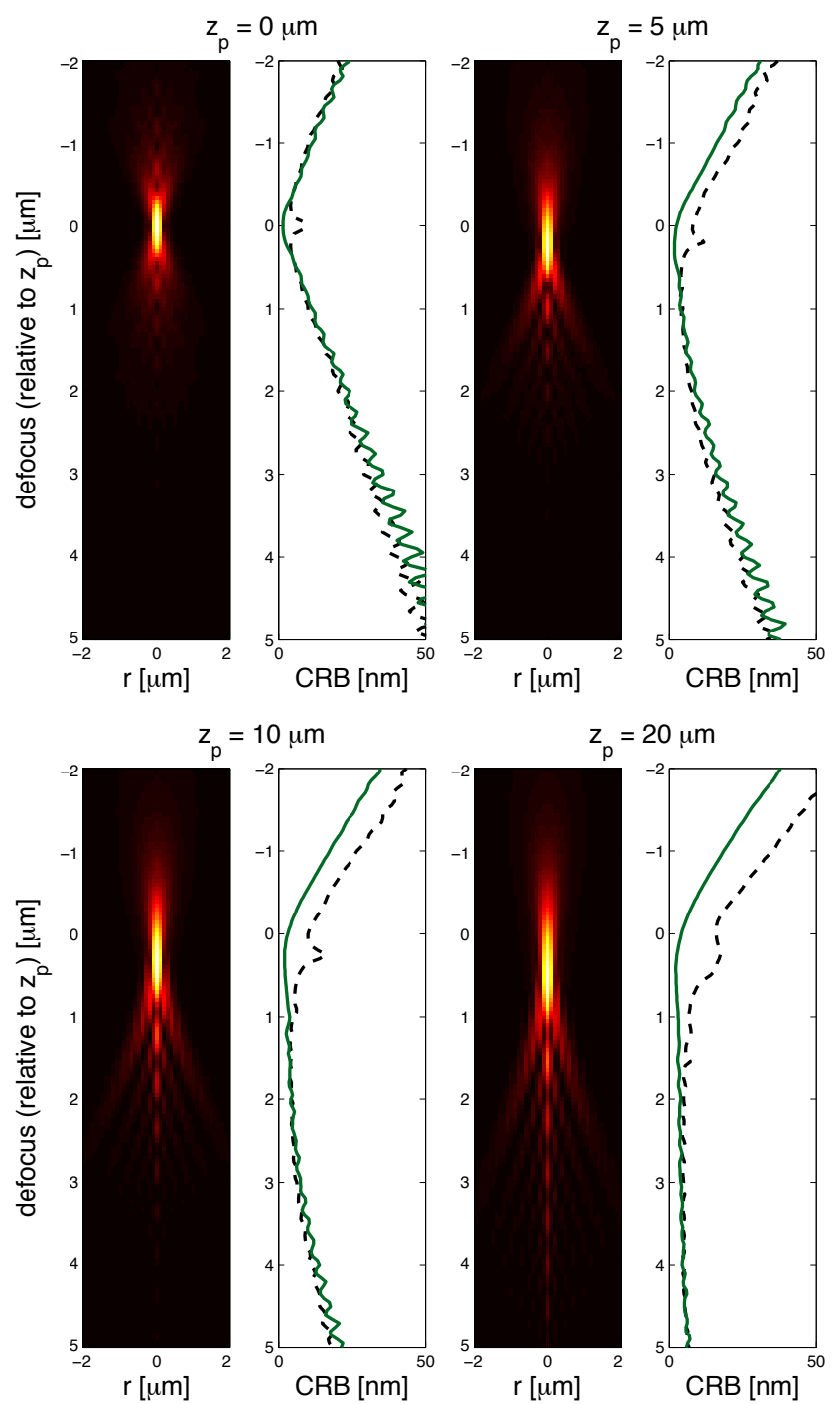

Fig. 1. xz-sections of the PSF shown together with the CRBs for $z_{p}$ (dashed black line) and $x_{p}$ (green line) for different axial positions $z_{p}$ of the particle (with $x_{p}$ centered between two pixels). The CRBs are shown as a function of defocus of the acquisition, for shot noise with $c=20, A=450$, and $\sigma_{b}^{2}=0$.

\subsection{Properties of the CRB}

Analyzing the behavior of the CRBs for specific experimental parameters gives some important insights into the imaging conditions required to maximize the localization accuracy.

The axial accuracy significantly improves when performed on out-of-focus acquisitions of particles that are located deeper into the specimen (see Fig. 1). In comparison, the localization accuracy in the acquisition plane evolves similarly, but is optimal where the intensity of the PSF is maximal, in which case the axial localization is usually poor. 

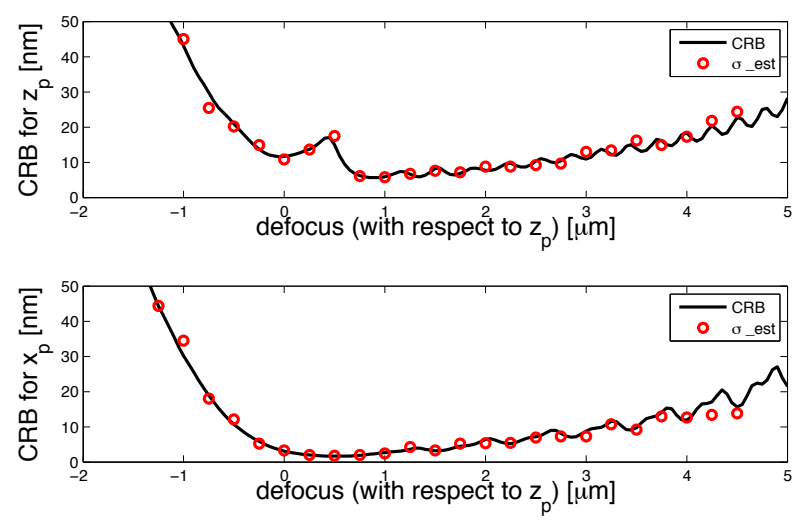

Fig. 2. Estimation results and CRBs for $z_{p}$ and $x_{p}$ as a function of defocus $\left(z-z_{p}\right)$, for a particle located at $\mathbf{x}_{p}=$ $(0.035,0.0,5.0) \mu m$. The estimator reaches the theoretical limit; for each focal position $(z)$, the estimation was performed on 50 different realizations of noise with $c=20$, $A=450$, and $\sigma_{b}^{2}=3$, yielding a $18.3 \mathrm{~dB}$ average SNR.

As the different plots show, the loss in accuracy for xylocalization under imaging conditions that are optimal for axial localization is small, and thus offers an easy compromise.

As Ober et al. have investigated, the xy-localization accuracy also varies as a function of the particle's position with respect to the CCD grid [4]. For 3-D localization experiments, our results show that the amount of defocus and the position of the particle within the specimen can be at least as important in determining the localization accuracy. Nevertheless, they can be optimized to some degree-especially when multiple particles are involved-using prior knowledge of the particles' distribution within the specimen [5].

\subsection{Estimation results}

In Fig. 2 we compare the standard deviation of the localization results using our estimator with the CRBs for $x_{p}$ and $z_{p}$. As the plots indicate, the estimator is optimal in the sense that it reaches the CRB. Its performance on experimental data is shown in Fig. 3. For each particle, a focal series separated by $100 \mathrm{~nm}$ was acquired over its entire visible range. To derive a ground truth for each particle's position, we performed a global fit of the unknown parameters using all available acquisitions, which also provided us with the acquisition positions $z$ required by the estimator, due to its reliance on a shiftvariant PSF. Further details on this calibration can be found in [5]. In the best cases, we achieved a localization accuracy of $12.8 \mathrm{~nm}$ axially and $7.15 \mathrm{~nm}$ in the acquisition plane.

A surprising and important aspect of our results comes from the comparison of the lateral and axial localization accuracies, where, contrary to the differences usually experienced in terms of resolution, the theoretical limits are very close.
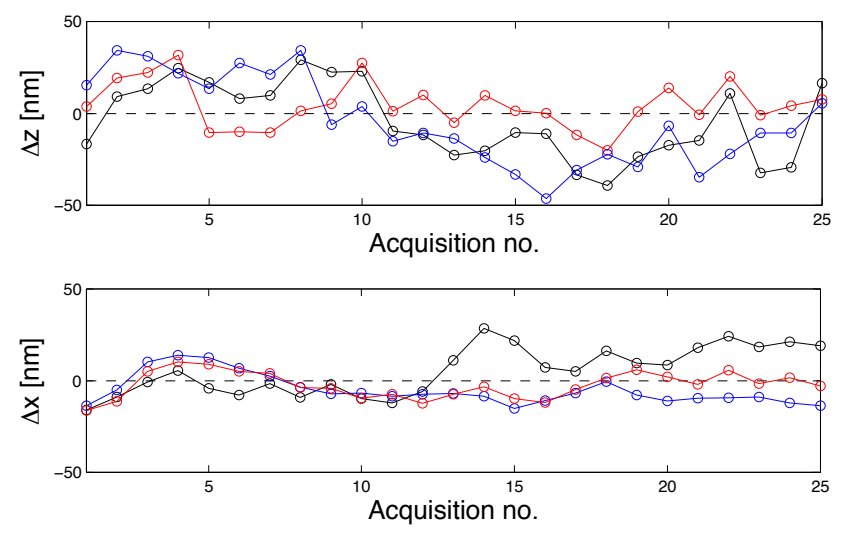

Fig. 3. Experimental localization results for three beads. The plots show the deviations $\Delta z=\hat{z}_{p}-z_{\text {ref }}$ and $\Delta x=\hat{x}_{p}-x_{\text {ref }}$, respectively, where $z_{\text {ref }}$ and $x_{\text {ref }}$ are the reference positions estimated using all acquisitions.

\section{REFERENCES}

[1] C. Vonesch, F. Aguet, J.-L. Vonesch, and M. Unser, "The colored revolution of bioimaging," IEEE Sig. Proc. Mag., vol. 23, no. 3, pp. 20-31, 2006.

[2] S. Hell and J. Wichmann, "Breaking the diffraction resolution limit by stimulated emission: stimulated-emission-depletion fluorescence microscopy," Opt. Lett., vol. 19, no. 11, pp. 780-782, 1994.

[3] N. Bobroff, "Position measurement with a resolution and noiselimited instrument," Rev. Sci. Instrum., vol. 57, pp. 1152-1157, 1986.

[4] R. J. Ober, S. Ram, and S. Ward, "Localization accuracy in single-molecule microscopy," Biophys J., vol. 86, pp. 11851200, 2004.

[5] F. Aguet, D. Van De Ville, and M. Unser, "A maximumlikelihood formalism for sub-resolution axial localization of fluorescent nanoparticles," Opt. Express, vol. 13, no. 26, pp. 10503-10522, 2005.

[6] E. Betzig, G. H. Patterson, R. Sougrat, O. W. Lindwasser, S. Olenych, J. S. Bonifacio, M. W. Davidson, J. LippincottSchwartz, and H. F. Hess, "Imaging intracellular fluorescent proteins at nanometer resolution," Science, vol. 313, no. 5793, pp. 1642-1645, 2006.

[7] S. Hell, G. Reiner, C. Cremer, and E. H. K. Stelzer, "Aberrations in confocal fluorescence microscopy induced by mismatches in refractive index," J. Microsc., vol. 169, pp. 391-405, 1993.

[8] P. Török and R. Varga, "Electromagnetic diffraction of light focused through a stratified medium," Appl. Opt., vol. 36, no. 11, pp. 2305-2312, 1997.

[9] S. F. Gibson and F. Lanni, "Experimental test of an analytical model of aberration in an oil-immersion objective lens used in three-dimensional light microscopy," J. Opt. Soc. Am. A, vol. 8, no. 10, pp. 1601-1613, 1991. 